\title{
Papers
}

\section{Screening INR deviation of local prothrombin time systems}

\author{
L Poller
}

\begin{abstract}
Aim-To assess the reliability of local international normalised ratios (INR) using a set of three international reference preparation (IRP) certified freeze dried plasmas.

Methods-55 centres in the United Kingdom and the Republic of Ireland participated; 36 centres employed coagulometers and 19 a manual prothrombin time technique, all with the same batch of routine commercial thromboplastin. The plasmas had certified INR with the manual technique using a thromboplastin IRP, and results were provided graphically to participants for self assessment. An INR deviation of more than $\pm 10 \%$ from the certified INR with any of the screening plasmas was regarded as unsatisfactory and clinically significant. Sets of 20 freeze dried plasmas were provided for local ISI calibrations and sets of seven freeze dried normals were provided for supplementary exercises where screening results were unsatisfactory.

Results-15 of 38 coagulometers, but only three of the 19 manual prothrombin time test techniques, gave unsatisfactory results. With 10 of the 15 unsatisfactory coagulometer results the problem was resolved by local ISI calibrations with plasma calibrant sets provided. Unsatisfactory results with manual technique in all four instances were corrected by substitution of the mean result with freeze dried normal plasmas provided.

Conclusions-The freeze dried plasma screening set was useful in detecting incorrect INR in a high proportion of coagulometer users and a smaller number of participants using the manual technique.

(f Clin Pathol 1998;51:356-359)
\end{abstract}

Keywords: INR; coagulometers; thromboplastin

The University of

Manchester, Stopford

Building, Oxford

Road, Manchester

M13 9PT, UK

L Poller

Correspondence to:

Professor L Poller.

Accepted for publication 11 February 1998 time standardisation scheme (1983), as mos laboratories have now replaced the manual technique on which this scheme was based by coagulometers. The international sensitivity index (ISI) of thromboplastins may be considerably modified by coagulometers and subsequent international normalised ratios (INR) derived from the ISI - that is, INR = prothrombin

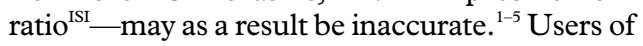
coagulometers therefore need a simple way of assessing the reliability of their INR, and guidance on the need for local ISI calibration of their prothrombin time system.

Freeze dried plasmas with certified manual prothrombin time values have been shown to be useful in correcting for local effects of coagulometers on the ISI of thromboplastins. ${ }^{5-7}$ A European Concerted Action on Anticoagulation (ECAA) multicentre study has shown that a set of 60 certified freeze dried plasmas gave a close approximation to the manual technique ISI, based on fresh plasmas from coumarin treated patients. ${ }^{8}$

In this report I describe a simple INR screening procedure using a small set consisting of three freeze dried test plasmas prepared for the study, representing a range of INR values. A multicentre exercise has been performed among coagulometer users and a group of centres employing the manual prothrombin time technique using the same brand of commercial thromboplastin. The freeze dried plasmas were certified with INR values with an international reference preparation (IRP) for thromboplastin. Their certified INR values and acceptable limits of performance were provided to participants graphically with the plasmas for self assessment. Results outside defined acceptable INR limits were regarded as showing the need for correction. For deviations greater than this, sets of freeze dried normal plasmas or sets of abnormal freeze dried plasma calibrants were provided to participants for follow up studies. In the present report I describe the findings in the 55 centre collaborative study.

\section{Methods}

REAGENTS

(a) Routine thromboplastin, Manchester Reagent (MR) batch 96/01 provided by Manchester Comparative Reagents Ltd (Manchester, UK). The batch was calibrated in full fresh plasma exercises based on fresh plasmas from 20 normal subjects and 60 coumarin treated patients in terms of both the human plain IRP (BCT/441) and 
WHO rabbit plain IRP (RBT/90), and gave ISI values of 1.09 and 1.07 respectively.

(b) Human plain thromboplastin IRP BCT/441 donated by the International Council for Standardisation in Haematology (ICSH, Custodian Laboratory, Dept of Pathological Sciences, University of Manchester, Manchester, UK). ISI $=1.04$.

(c) Rabbit plain thromboplastin IRP RBT/90 donated by WHO Biologicals (Geneva, Switzerland). ISI $=1.00$.

(d) INR screening plasmas. The set of three plasmas was prepared for the study by artificial depletion of normal human donor plasma. They were certified in quadruplicate prothrombin time tests by six experienced operators. The mean prothrombin time value was extrapolated as an INR using the ICSH human plain IRP (BCT/441) and the conventional formula given above. Mean INR of 2.0, 2.8, and 3.35 were thus obtained.

(e) Freeze dried normal plasmas. These were prepared for use where necessary in the follow up studies to check the reliability of the local mean normal prothrombin time (MNPT) where the screening plasma results were within the limits but the prothrombin ratios were not. The normal freeze dried plasmas were obtained from normal healthy donors. Seven were provided to maintain the similar normal/ abnormal balance to conventional fresh plasma ISI calibrations, where 20 normals are combined with 60 abnormal plasmas to provide the calibration slope. In this study 20 abnormal freeze dried plasma calibrants were employed for local ISI calibrations; thus the number of normal plasmas was proportionately reduced.

(f) Sets of abnormal plasma calibrants. Sets of 20 plasmas were provided for local ISI calibration. It has been shown in a European Concerted Action on Anticoagulation study that 20 artificially depleted freeze dried plasmas, when reliably certified with prothrombin time values with a thromboplastin IRP using the manual technique, give a reliable and precise ISI calibration. ${ }^{9}$ The sets of 20 freeze dried artificially depleted plasmas spanned the 1.5 to 4.5 INR range. They were donated by Manchester Comparative Reagents Ltd, and certified with the thromboplastin IRP and the manual prothrombin time technique by the same group of six operators as were the INR screening set and normal freeze dried plasmas. They were provided for follow up studies where a local ISI calibration appeared necessary.

INSTRUCTIONS TO PARTICIPANTS

Participants were routine users of the MR commercial thromboplastin and had agreed in advance to participate in the INR screening study.

The three INR screening plasma vials were to be reconstituted with $0.5 \mathrm{ml}$ distilled water, mixed gently, and left at room temperature for
Table 1 Coagulometers used by participants

\begin{tabular}{lll}
\hline Coagulometer & Total & Satisfactory \\
\hline All & $\mathbf{3 8}$ & $\mathbf{2 3}$ \\
(1) ACL & 11 & 3 \\
(2) Coag-a-mate & 6 & 5 \\
(3) Sysmex & 5 & 5 \\
(4) Stago & 1 & 1 \\
(5) Biomerieux Option 4 & 2 & 1 \\
(6) Amelung KC & 9 & 7 \\
(7) Electra & 1 & 0 \\
(8) Behring Fibrin Timer & 1 & 0 \\
(9) Cobas Fibro & 1 & 1 \\
(10) Helena & 1 & 0 \\
\hline
\end{tabular}

a least 10 minutes. Prothrombin times were to be performed in duplicate with each of the reconstituted plasmas and the customary local prothrombin time technique.

For each plasma, INR was calculated by participants as follows:

$\mathrm{INR}=\left|\frac{\text { mean duplicate PT }}{\mathrm{MNPT}}\right|^{\text {ISI }^{\star}}$

${ }^{\star}$ ISI used routinely by participant

Centres were provided with a chart giving the certified INR values for the screening plasmas (see figs 1-4) and their acceptable limits $( \pm 10 \%$ from mean certified INR). They were asked to insert their own INR with the three screening plasmas and return the set of results within three weeks.

If the INR of any of the three plasmas deviated by more than the $\pm 10 \%$ from the certified INR the prothrombin time test on the plasma was to be repeated. If on repeat testing an unsatisfactory result was again obtained, participants were asked to check their MNPT by determining the mean value from the seven freeze dried normals supplied for the supplementary exercise. If after insertion of the new normal value any INR was still beyond the $10 \%$ limit a local ISI calibration with the set of 20 freeze dried plasma calibrants was to be performed.

\section{Results}

Fifty five invited centres (51 in the United Kingdom and four in the Republic of Ireland) returned results. Thirty six were coagulometer users of which two tested two instruments. Nineteen centres employed the manual prothrombin time technique.

Of the coagulometers, 23 of the 38 gave satisfactory results ( $<10 \%$ INR deviation) from the certified INR, whereas of the 19 manual techniques 16 were satisfactory with all three certified INR plasmas. The number of users of individual coagulometers is given in table 1 , which shows the proportion with satisfactory INR with all three test plasmas. Of the 15 instruments which gave excessive deviation with at least one plasma, INR problems in 10 were resolved by local ISI calibration using the set of 20 abnormal freeze dried plasmas, while four were corrected by a check on the normal (MNPT) value using the freeze dried normal plasmas (table 2). One coagulometer problem remained unresolved as the centre did not wish to pursue matters. 
Table 2 Unsatisfactory screening results and method of correction

\begin{tabular}{|c|c|c|c|c|}
\hline $\begin{array}{l}\text { (A) Coagulometers } \\
\text { Instrument }\end{array}$ & $n$ & $\begin{array}{l}\text { No of plasmas } \\
\text { out of range }\end{array}$ & $\begin{array}{l}\text { Resolved by } \\
\text { local calibration }\end{array}$ & Normal check \\
\hline ACL & 8 & $24 / 24$ & 7 & 0 \\
\hline Coag-a-mate & 1 & $3 / 3$ & - & 1 \\
\hline $\mathrm{KC}$ & 2 & $4 / 6$ & - & 2 \\
\hline Helena & 1 & $1 / 3$ & - & 1 \\
\hline Electra & 1 & $1 / 3$ & 1 & - \\
\hline Biomerieux Option 4 & 1 & $3 / 3$ & 1 & - \\
\hline Behring Fibrin Timer & 1 & $3 / 3$ & 1 & - \\
\hline \multicolumn{5}{|l|}{ (B) Manual technique } \\
\hline Total & Satisfactory & $\begin{array}{l}\text { No of plasmas } \\
\text { out of range }\end{array}$ & $\begin{array}{l}\text { Resolved by } \\
\text { local calibration }\end{array}$ & Normal check \\
\hline 19 & 16 & $5 / 9$ & - & 3 \\
\hline
\end{tabular}

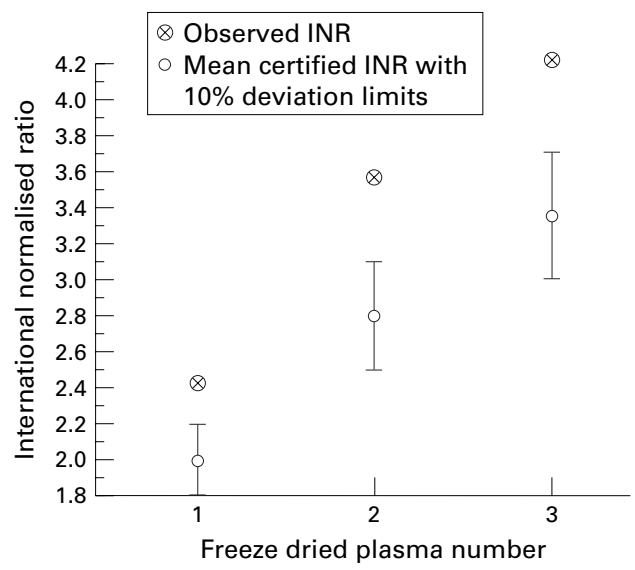

Figure 1 Example of unsatisfactory INR screening test with a coagulometer.

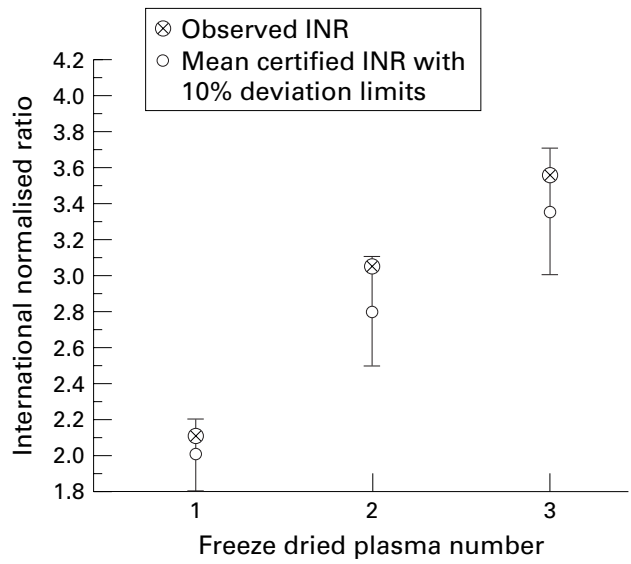

Figure 2 Screening results after local ISI calibration on coagulometer.

Of the 19 users of the manual technique, 16 gave satisfactory results. Of the three remaining manual prothrombin time centres with high deviation with at least one plasma, five of nine of the INR screening plasmas fell outside the $10 \%$ limit. In all three centres the problem was found to be the result of an incorrect MNPT, and a local ISI calibration was not found necessary.

Examples of satisfactory and unsatisfactory INR screening results with manual prothrombin time tests and coagulometers are given in figs 1-4. The examples given are typical ones and are by no means extreme examples of the unsatisfactory performance in the screening exercise.

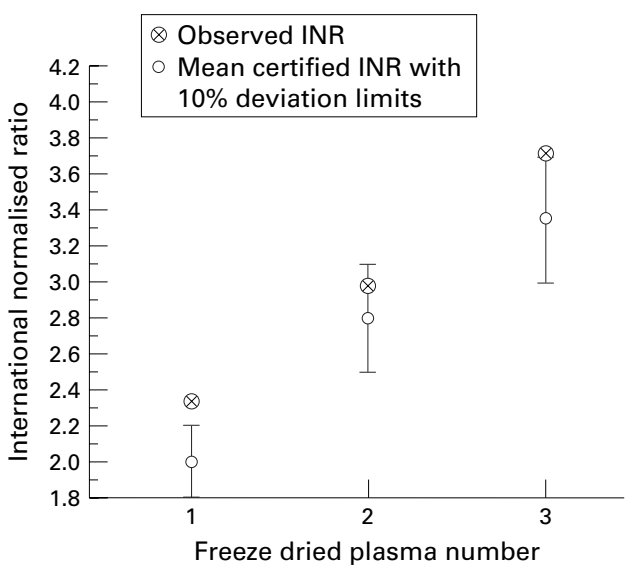

Figure 3 Example of unsatisfactory screening results with manual technique.

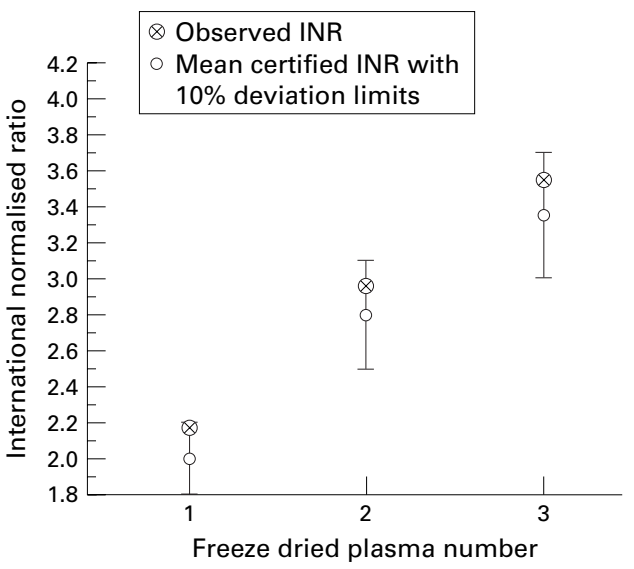

Figure 4 Screening results from same centre with manual technique after correction of normal.

\section{Discussion}

The United Kingdom NEQAS scheme recently highlighted significant differences which occur between laboratories in INR estimation.

The present multicentre study was the first experience of an INR screening procedure for local prothrombin time systems. A deviation of $\pm 10 \%$ from the certified INR value has been arbitrarily defined as the limit of satisfactory performance following the example of the ECAA study, where it was felt that this was significant in terms of clinical response to an anticoagulant dose..$^{10}$

The use of an INR screening kit consisting of three certified freeze dried plasmas has confirmed that the problems of local INR deviation are mainly the result of an effect on the ISI of the thromboplastin by coagulometers. Unsatisfactory results were recorded with a considerable proportion of these instruments (15 of 38 screenings). In contrast only three of 19 manual prothrombin time test procedures gave one or more results with the three test plasmas outside the $10 \%$ deviation limit. In none of these instances with the manual prothrombin time technique did a local ISI calibration prove necessary, as the fault was found to be in the MNPT used to obtain the prothrombin ratios from which INR are derived.

Of the 15 coagulometers which gave one or more unsatisfactory INR results with the screening plasmas, a check on the MNPT was 
necessary in only four of the 15 instances, while a local ISI calibration using the sets of 20 freeze dried plasma calibrants was required in the others. However, not all coagulometers produced a dramatic difference in the INR from the manually certified values, as shown in table 1. The largest number of unsatisfactory results with the thromboplastin appeared to be with the coagulometer used in the largest number in the United Kingdom laboratories (ACL). Only three of $11 \mathrm{ACL}$ instruments gave satisfactory screening results, whereas 20 of 27 of the other coagulometers gave satisfactory results. The marked effect of the ACL coagulometer on the ISI of Manchester Reagent has been demonstrated previously in a multicentre national study $^{5}$ and similarly on the ISI of two other routine commercial thromboplastin reagents in an international collaborative study. ${ }^{7}$

Although the results of the present study are based on one commercial thromboplastin reagent, it appears a reasonable assumption that they would not have been dramatically altered if a different choice of a routine reagent had been made. Where routine reagents may not be well calibrated by manufacturers or alternatively differ greatly in ISI from the relevant IRP, it can be reasonably assumed that the possibility of INR deviation may be greater and that the benefit of the INR screening procedure will therefore be even more marked.

The results prove the value of the screening kit with freeze dried plasmas certified with INR in terms of a thromboplastin IRP. They show the need for local INR correction in a considerable proportion (15 of 38) of coagulometer users. The simple screening procedure described also supported the need for reassessment of the local normal value for ISI correction with a smaller proportion of coagulometer users compared with the manual technique, where the small number of unsatisfac- tory results were all a result of errors in the normal.

It is not recommended, however, that the three freeze dried plasmas should be used to provide a local ISI value, as it has been shown that a minimum of 20 plasmas of this type is required for a reliable ISI result. ${ }^{10}$

Thanks are due to the directors and technical staff of the participant centres in the United Kingdom and the Republic of Ireland for their cooperation in assessing the value of the freeze dried plasma screening procedure, and to Manchester Thrombosis Research Foundation and the Biomed 1 Programme of the bosis Research Foundation and the Biomed 1 Programme of the
European Commission (grant number PL931349) for financial support.

1 D'Angelo A, Seveso MP, D'Angelo SV, et al. Comparison of two automated coagulometers and the manual tilt tube method for the determination of prothrombin time. Am $\mathcal{F}$ Clin Pathol 1989;92:321-8.

2 Poller L, Thomson JM, Taberner DA. Effect of automation on the prothrombin time test in NEQAS surveys. $\mathcal{F}$ Clin Pathol 1989;42:97-100.

3 van Rijn JLML, Schmidt Nico A, Rutten W. Correction of instrument and reagent based differences in determination of the international normalised ratio (INR) for monitoring anticoagulant therapy. Clin Chem 1989;355:840-3.

4 Ray MJ, Smith IR. The dependence of the International Ray MJ, Smith IR. The dependence of the International Sensitivity Index on the coagulometer used to perfor
prothrombin time. Thromb Haemost 1990;63:424-9.

5 Clarke K, Taberner DA, Thomson JM, et al. Assessment of value of calibrated lyophilized plasmas to determine International Sensitivity Index for coagulometers. F Clin Pathol 1992;45:58-60.

6 van den Besselaar AMHP \& Peters RHM. Multicentre evaluation of the Thrombotest International Sensitivity Index used with a steel ball coagulometer. F Clin Patho

7 Poller L, Triplett DA, Hirsh J, et al. The value of plasma calibrants in correcting coagulometer effects on International Normalized Ratios-an international multicenter study. Am 7 Clin Pathol 1995;103:358-65.

8 European Concerted Action on Anticoagulation (ECAA), Poller L, van den Besselaar AHMP, Jespersen J, et al. A comparison of artificially depleted, lyophilized coumarin and fresh coumarin plasmas in thromboplastin calibration. Brf Haematol (in press).

9 Kitchen S, Walker ID, Woods TAL, et al. Thromboplastin related differences in the determination of international normalised ratio: a cause for concern? Thromb Haemost 1994;72:426-9.

10 European Concerted Action on Anticoagulation (ECAA), Poller L, Barrowcliffe TW, van den Besselaar AHMP, et al. Minimum lyophilized plasma requirement for ISI calibration. Am f Clin Pathol 1998;109:196-204. 